\title{
Supernova neutrino scattering off Gadolinium odd isotopes in water Cherenkov detectors.
}

\author{
Paraskevi C. Divari \\ Department of Physical Sciences and Applications, \\ Hellenic Military Academy, Vari 16673, Attica, Greece
}

\begin{abstract}
In this work the supernova neutrino(SN) charged-current interactions with Gd odd isotopes $(\mathrm{A}=155$ and 157$)$ are studied. We use measured spectra and the quasiparticle-phonon model (MQPM) to calculate the charged current response of odd Gd isotopes to supernova neutrinos. Flux-averaged cross sections are obtained considering quasi-thermal neutrino spectra.

PACS numbers: 26.50.+x, 13.15.+g, 25.30.Pt, 28.20.-V
\end{abstract}

\section{INTRODUCTION}

Neutrino physics has become the main focus of astrophysics, particle physics and nuclear physics. The Galaxy Supernova only happens once every 30 years. Various detectors must be prepared to detect all flavors of neutrinos well to understand the physics and astrophysics of nuclear collapsed supernova. Neutrino oscillations provide solid evidence for phenomena other than standard model physics, and the experimental work used to detect weakly interacting particles is more powerful than ever. Using current detectors, it is easy to measure supernovae $\bar{\nu}_{e}$ and $\nu_{x}$ via inverse beta decay (IBD) and elastic scattering on protons, respectively [ 1 [3]. Detecting supernova neutrinos is an indispensable method for testing supernova and neutrino physics, but the yield is small, and the background from other channels is also very large.

A number of detectors like water Cherenkov detectors (WCDs) [4 [6], have been used in various neutrino detection experiments. The neutrino detection in WCDs is based on the detection of Cherenkov radiation. A charged particle moving through at a speed greater than the speed of light in the media will generate Cherenkov radiation. The radiation is emitted at an angle $\theta$ to the direction of travel, which are then detected by the photomultiplier tubes (PMTs) lined in the inner walls of the detector. Neutrinos in water can be detected thanks to several reactions. The most important are the IBD, the elastic scattering on electrons and the neutral current scattering on oxygen ${ }^{16} \mathrm{O}$.

In an inverse beta decay, an electron antineutrino scattering from a proton, creates a positron and a neutron. The positron undergoes a prompt matter-antimatter annihilation emitting light and the neutron capture on hydrogen nucleus release not detectable $2.2 \mathrm{MeV}$ gamma cascades (energy threshold in Super-Kamiokande(SK) about $5 \mathrm{MeV}$ ). The sensitivity of the detectors can be enhanced including additives, such as gadolinium-based salt compounds, in water that essentially reduces background signals [7]. The neutron capture by gadolinium (Gd) results in $8 \mathrm{MeV}$ gamma cascades with temporal and spatial coincidence with the positron from the initial interaction. This delayed neutron capture by Gd and higher gamma emission energy compared to neutron capture by hydrogen nucleus, creates a unique signature and improves background reduction to an antineutrino event and thus allows antineutrino detection in WCDs through IBD. In SK, which is a 32 ktons (fiducial) WCD, it has be found that the inclusion of $\mathrm{GdCl}_{3} \mathrm{salt}$ ( $0.2 \%$ in weight) to SK, $\sim 90 \%$ of the IBD events could be tagged [7, 8]. The remaining IBD events as well as the $\bar{\nu}_{e}$ absorption events on ${ }^{16} \mathrm{O}$ can then be statistically subtracted from the remaining signal.

In Ref. 9] we payed special attention on calculations of charged current (CC) neutrino/antineutrino-Gd cross sections at neutrino energies below $80 \mathrm{MeV}$, considering the most abundant even isotopes of Gadolinium that is, isotopes with mass number $\mathrm{A}=156,158$ and 160 (20.47\%, 24.84\% and 21.86\% abundant, respectively). In this article, 
we have expanded this calculation list and calculated the cross-sections of charged current neutrinos and antineutrinos scattering off the stable odd gadolinium isotopes, ${ }^{155} \mathrm{Gd}$ and ${ }^{157} \mathrm{Gd}$ (14.8\% and $15.6 \%$ abundant, respectively). The energy of impinging neutrinos is related to supernova neutrinos while the corresponding nuclear matrix elements have been calculated in the framework of microscopic quasiparticle-phonon model.

\section{BRIEF DESCRIPTION OF THE FORMALISM}

The standard model effective Hamiltonian in the charged current reactions

$$
\begin{aligned}
& (A, Z)+\nu_{e} \rightarrow(A, Z+1)+e^{-} \\
& (A, Z)+\bar{\nu}_{e} \rightarrow(A, Z-1)+e^{+}
\end{aligned}
$$

can be written

$$
\mathcal{H}=\frac{G_{F} \cos \theta_{c}}{\sqrt{2}} j_{\mu}(\mathbf{x}) J^{\mu}(\mathbf{x})
$$

$\mathrm{A}(\mathrm{Z})$ represents the mass(proton) number of a nucleus, respectively. Here $G_{F}=1.1664 \times 10^{-5} \mathrm{GeV}^{-2}$ denotes the Fermi weak coupling constant and $\theta_{c} \simeq 13^{\circ}$ is the Cabibbo angle. According to V-A theory, the leptonic current takes the form 10 13]

$$
j_{\mu}=\bar{\psi}_{\nu_{\ell}}(x) \gamma_{\mu}\left(1-\gamma_{5}\right) \psi_{\nu_{\ell}}(x)
$$

where $\psi_{\nu_{\ell}}$ are the neutrino/antineutrino spinors. The hadronic current of vector, axial-vector and pseudo-scalar components is written as

$$
\begin{array}{r}
J_{\mu}=\bar{\Psi}_{N}\left[F_{1}\left(q^{2}\right) \gamma_{\mu}+F_{2}\left(q^{2}\right) \frac{i \sigma_{\mu \nu} q^{\nu}}{2 M_{N}}+F_{A}\left(q^{2}\right) \gamma_{\mu} \gamma_{5}\right. \\
\left.+F_{P}\left(q^{2}\right) \frac{1}{2 M_{N}} q_{\mu} \gamma_{5}\right] \Psi_{N}
\end{array}
$$

( $M_{N}$ stands for the nucleon mass, $\Psi_{N}$ denotes the nucleon spinors and $q^{2}$, the square of the four-momentum transfer). By the conservation of the vector current (CVC), the vector form factors $F_{1,2}\left(q^{2}\right)$ can be written in terms of the proton and neutron electromagnetic form factors [14]. The axial-vector form factor $F_{A}\left(q^{2}\right)$ is assumed to be of dipole form [15] while the pseudoscalar form factor $F_{P}\left(q^{2}\right)$ is obtained from the Goldberger-Treiman relation [10].

In the convention we used in the present work the square of the momentum transfer, is written as

$$
q^{2}=q^{\mu} q_{\mu}=\omega^{2}-\mathbf{q}^{2}=\left(\varepsilon_{i}-\varepsilon_{f}\right)^{2}-\left(\mathbf{p}_{i}-\mathbf{p}_{f}\right)^{2},
$$

where $\omega=\varepsilon_{i}-\varepsilon_{f}$ is the excitation energy of the final nucleus. $\varepsilon_{i}\left(\mathbf{p}_{i}\right)$ denotes the energy(3-momenta) of the incoming neutrino/antineutrino and $\varepsilon_{f}\left(\mathbf{p}_{f}\right)$ those of the outgoing electron/positron, respectively. The charged-current neutrino/antineutrino-nucleus cross section is written as 11]

$$
\begin{gathered}
\sigma\left(\varepsilon_{i}\right)=\frac{2 G_{F}^{2} \cos ^{2} \theta_{c}}{2 J_{i}+1} \sum_{f}\left|\mathbf{p}_{f}\right| \varepsilon_{f} \int_{-1}^{1} d(\cos \theta) F\left(\varepsilon_{f}, Z_{f}\right) \\
\times\left(\sum_{J=0}^{\infty} \sigma_{C L}^{J}(\theta)+\sum_{J=1}^{\infty} \sigma_{T}^{J}(\theta)\right)
\end{gathered}
$$

$\theta$ denotes the lepton scattering angle. The summations in Eq. (5) contain the contributions $\sigma_{C L}^{J}$, for the Coulomb $\widehat{\mathcal{M}}_{J}$ and longitudinal $\widehat{\mathcal{L}}_{J}$, and $\sigma_{T}^{J}$, for the transverse electric $\widehat{\mathcal{T}}_{J}^{e l}$ and magnetic $\widehat{\mathcal{T}}_{J}^{\text {mag }}$ multipole operators defined as in Ref. [16]. These operators include both polar-vector and axial-vector weak interaction components. 
TABLE I: Adjusted (Adj) single-particle energies together with the Woods-Saxon (WS) energies (in MeV) for the neutron $(n)$ and proton $(p)$ orbitals.

\begin{tabular}{|c|c|c|c|c|c|c|}
\hline orbital & ${ }^{154} \mathrm{Gd}$ & & ${ }^{156} \mathrm{Gd}$ & & ${ }^{158} \mathrm{Gd}$ & \\
\hline & WS & Adj & WS & $\mathrm{Adj}$ & WS & $\mathrm{Adj}$ \\
\hline $\mathrm{n} 2 \mathrm{p}_{1 / 2}$ & & & -3.5015 & -4.8435 & & \\
\hline $\mathrm{n} 2 \mathrm{p}_{3 / 2}$ & -4.5188 & -5.7000 & -4.4950 & -5.5000 & -4.4727 & -5.8000 \\
\hline $\mathrm{n} 1 f_{5 / 2}$ & & & -3.6510 & -6.3407 & -3.6580 & -4.4000 \\
\hline $\mathrm{n} 1 f_{7 / 2}$ & -6.4707 & -4.6800 & -6.4352 & -3.9263 & -6.4017 & -6.5000 \\
\hline $\mathrm{nOh}_{9 / 2}$ & & & -5.6821 & -3.8911 & -5.7082 & -4.2000 \\
\hline $\mathrm{nOh}_{11 / 2}$ & & & -11.0539 & -1.4720 & & \\
\hline $\mathrm{p} 1 d_{3 / 2}$ & -3.9148 & -6.6000 & -4.4795 & -6.7000 & -5.0446 & -6.0000 \\
\hline $\mathrm{p} 1 \mathrm{~d}_{5 / 2}$ & -6.2413 & -7.7000 & & & -7.3505 & -7.9000 \\
\hline $\mathrm{p} 0 \mathrm{~g}_{7 / 2}$ & & & & & -8.1939 & -7.2000 \\
\hline $\mathrm{p} 0 \mathrm{~g}_{9 / 2}$ & & & & & -12.7976 & -10.0000 \\
\hline $\mathrm{p} 0 \mathrm{~h}_{11 / 2}$ & & & & & -6.0117 & -12.0000 \\
\hline
\end{tabular}

\section{MQPM}

The microscopic quasiparticle-phonon model (MQPM ) is a microscopic nuclear model which treats the structure of the odd-A nuclei. The Hamiltonian can be written in the form

$$
H=\sum_{\alpha} E_{a} a_{\alpha}^{\dagger} a_{\alpha}+H_{22}+H_{40}+H_{04}+H_{31}+H_{13}
$$

were $E_{\alpha}$ are the quasiparticle energies and other terms of the hamiltonian are normal ordered parts of the residual interaction labeled according to the number of quasiparticle creation and annihilation operators which they contain.

The $H_{22}, H_{40}$ and $H_{04}$ parts of the quasiparticle Hamiltonian are treated in the BCS and quasiparticle random-phase approximation (QRPA) framework and leads to definition of the quasiparticles and the excitation (phonon) spectrum of the doubly-even reference nucleus [17]. The $H_{31}$ and $H_{13}$ parts are then diagonalized in the quasiparticle-phonon basis and the eigenvectors represent the spectrum of the odd-A nucleus.

The approximate ground state of the even-even reference nucleus is obtained from a BCS calculation, where quasiparticle energies and occupation factors $u_{a}$ and $v_{a}$ are obtained from the Bogoliubov-Valatin transformation to quasiparticles

$$
\begin{aligned}
& a_{\mu}^{\dagger}=u_{\mu} c_{\mu}^{\dagger}-v_{\mu} \tilde{c}_{\mu} \\
& \tilde{a}_{\mu}^{\dagger}=u_{\mu} \tilde{c}_{\mu}^{\dagger}+v_{\mu} c_{\mu}
\end{aligned}
$$

where $\tilde{a}_{\mu}^{\dagger}=a_{-\mu}^{\dagger}(-1)^{j+m}$ and $\tilde{c}_{\mu}^{\dagger}=c_{-\mu}^{\dagger}(-1)^{j+m}$. In Eq. (7) $c_{\mu}^{\dagger}$ is the particle creation operator and $\tilde{c}_{\mu}^{\dagger}$ denotes the time-reversed particle annihilation operator.

In the QRPA the creation operator for an excited state (QRPA phonon) has the form

$$
Q_{\omega}^{\dagger}=\sum_{a \leq a^{\prime}}\left[X_{a a^{\prime}}^{\omega} A^{\dagger}\left(a a^{\prime} ; J_{\omega} M\right)-Y_{a a^{\prime}}^{\omega} \tilde{A}\left(a a^{\prime} ; J_{\omega} M\right)\right]
$$


where the quasiparticle pair creation and annihilation operators are defined as $A^{\dagger}\left(a a^{\prime} ; J M\right)=\sigma_{a a^{\prime}}^{-1}\left[a_{a}^{\dagger} a_{a^{\prime}}^{\dagger}\right]_{J M}$, $\tilde{A}\left(a a^{\prime} ; J M\right)=\sigma_{a a^{\prime}}^{-1}\left[\tilde{a}_{a} \tilde{a}_{a^{\prime}}\right]_{J M}$ and $\sigma_{a a^{\prime}}=\sqrt{1+\delta_{a a^{\prime}}}$. Here the greek indices $\omega$ denote phonon spin $J$ and parity $\pi$. Furthermore, they contain an additional quantum number $k$ enumerating the different QRPA roots for the same angular momentum and parity. Thus $\omega=\left\{J_{\omega}, \pi_{\omega}, k_{\omega}\right\}$.

In the MQPM a state with angular momentum $j$ and projection $m$ in an odd-A nucleus is then created by using the following operator

$$
\Gamma_{k}^{\dagger}(j m)=\sum_{n} D_{n}^{k} a_{n j m}^{\dagger}+\sum_{\omega \alpha} D_{\alpha \omega}^{k}\left[a_{\alpha}^{\dagger} Q_{\omega}^{\dagger}\right]_{j m}
$$

The MQPM states with one dominant amplitude $D_{n}^{k}$ are called one-quasiparticle-like and states with one,few or many important amplitudes $D_{\alpha \omega}^{k}$ are called three-quasiparticle-like states. For more details about the MQPM see Ref [18].

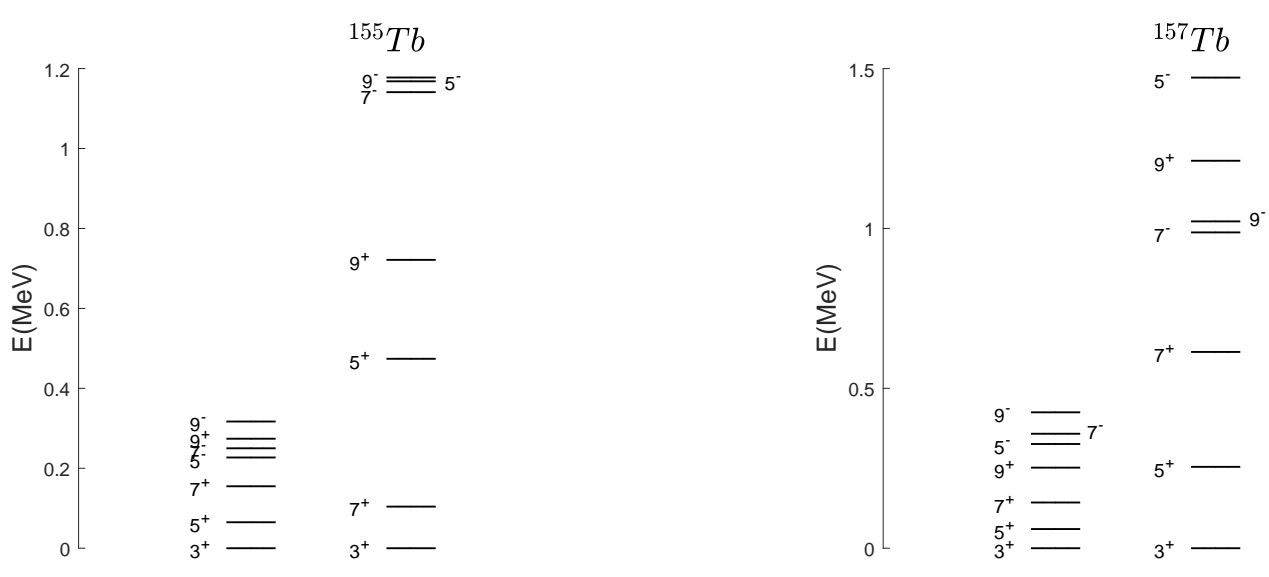

FIG. 1: (Color on line) Experimental (left) [19, 20] and theoretical (right) spectra of ${ }^{155} \mathrm{~Tb}$ and ${ }^{157} \mathrm{~Tb}$

\section{RESULTS}

For the reactions ${ }^{A} G d\left(\bar{\nu}_{e}, e^{+}\right){ }^{A} E u, \mathrm{~A}=155,157$ the initial sates of ${ }^{155} \mathrm{Gd}\left({ }^{157} \mathrm{Gd}\right)$ are taken as neutron hole states of the reference nucleus ${ }^{156} \mathrm{Gd}\left({ }^{158} \mathrm{Gd}\right)$ while those of ${ }^{155} \mathrm{Eu}\left({ }^{157} \mathrm{Eu}\right)$ as proton hole states. On the other hand for the reactions ${ }^{A} G d\left(\nu_{e}, e^{-}\right){ }^{A} T b$ the initial states of ${ }^{155} \mathrm{Gd}\left({ }^{157} \mathrm{Gd}\right)$ are taken as neutron states of the reference nucleus ${ }^{154} \mathrm{Gd}\left({ }^{156} \mathrm{Gd}\right)$, while those of ${ }^{155} \mathrm{~Tb}\left({ }^{157} \mathrm{~Tb}\right)$ as proton ones.

The wave functions of initial and final states are computed by using the microscopic quasiparticle phonon model (MQPM) ([18]). The active model space for protons consists of the complete oscillator shells $4 \hbar \omega$ and $5 \hbar \omega$ while for neutrons the oscillator shells are $5 \hbar \omega$ and $6 \hbar \omega$. The corresponding single particle energies (s.p.e) were produced by the well known Coulomb corrected Woods-Saxon potential adopting the parameters of Bohr and Mottelson [21]. The pairing interaction between the nucleons can be adjusted by solving the corresponding BCS equations [9].

In addition, adjustments of some single-particle energies, by comparison with spectra of the neighboring odd nuclei, were done. The adjustments made are shown in Table@. The two-body matrix elements were obtained from the Bonn one-boson-exchange potential applying the G-matrix techniques [22]. The phonon basis contains all QRPA states having angular momentum $J^{\pi} \leq 6^{ \pm}$and excitation energy $E \leq 10 \mathrm{MeV}$. As an example the calculated energy spectra 
of low-lying states up $1.5 \mathrm{MeV}$ for ${ }^{155,157} \mathrm{~Tb}$ isotopes together with the experimental ones [19, 20] are presented in Fig. 1

In Fig. 2 (a) and (b) we display the numerical results of the total scattering cross section $\sigma\left(E_{\nu}\right)$ given by Eq. (5) as a function of the incoming neutrino energy $E_{\nu}$ for the reactions ${ }^{A} G d\left(\nu_{e}, e^{-}\right){ }^{A} T b$ and ${ }^{A} G d\left(\bar{\nu}_{e}, e^{+}\right){ }^{A} E u, A=155,157$ respectively, along with the results concerning the even isotopes $\mathrm{A}=156,158,160$ taken from Ref. [9]. In Fig. 2 also shown are the total cross sections of $G d\left(\nu_{e}, e^{-}\right) T b$ (solid line) and $G d\left(\bar{\nu}_{e}, e^{+}\right) E u$ (dashed line) for natural Gadolinium(the natural abundance of each isotope has been taken into account). The normalized differential cross sections

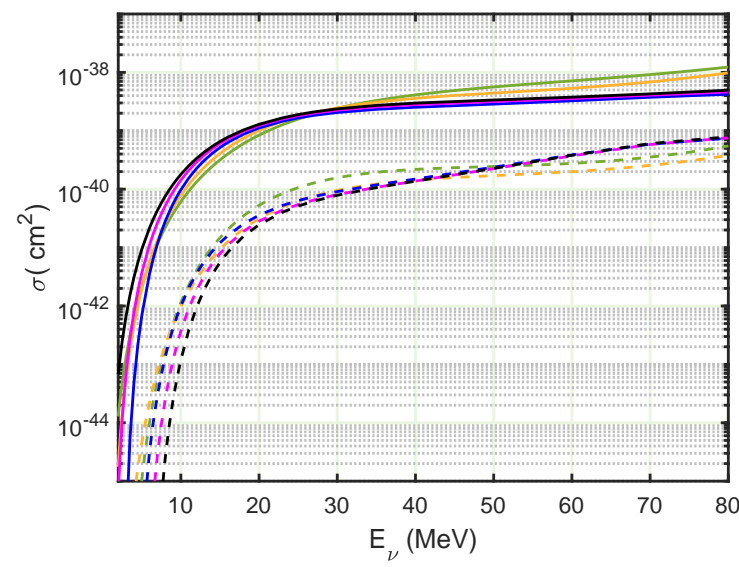

a)

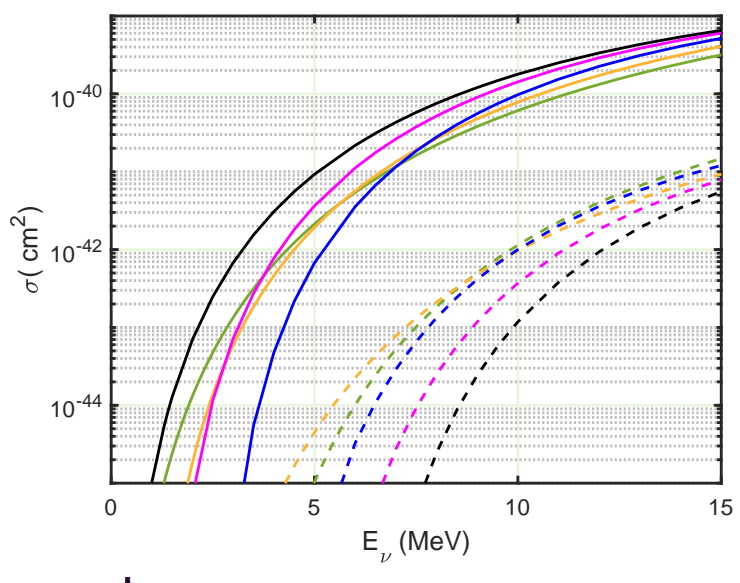

b)

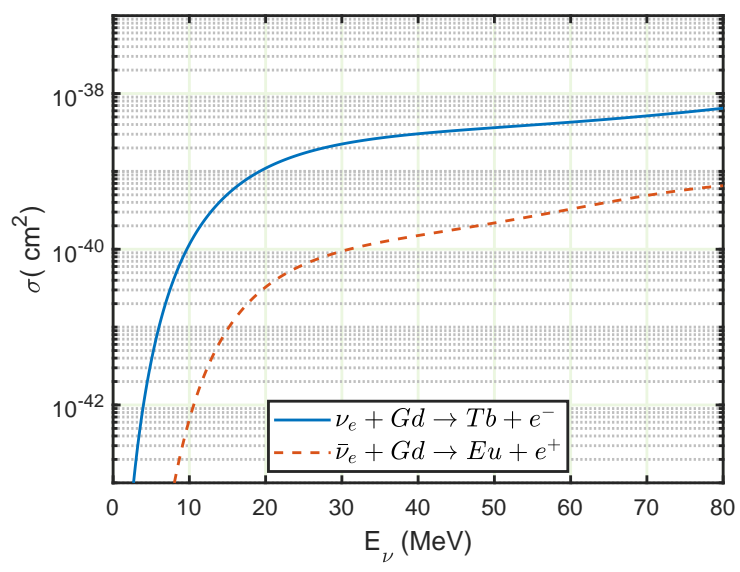

$-\nu_{e}+{ }^{157} \mathrm{Gd} \rightarrow \rightarrow^{157} \mathrm{~Tb}+e^{-}$
$-\nu_{e}+{ }^{155} \mathrm{Gd} \rightarrow{ }^{155} \mathrm{~Tb}+e^{-}$
$---\bar{\nu}_{e}+{ }^{157} \mathrm{Gd} \rightarrow \rightarrow^{157} \mathrm{Eu}+e^{+}$
$---\bar{\nu}_{e}+{ }^{155} \mathrm{Gd} \rightarrow \rightarrow^{155} \mathrm{Eu}+e^{+}$
$-\nu_{e}+{ }^{156} \mathrm{Gd} \rightarrow \rightarrow^{156} \mathrm{~Tb}+e^{-}$
$-\nu_{e}+{ }^{158} \mathrm{Gd} \rightarrow \rightarrow^{158} \mathrm{~Tb}+e^{-}$
$-\nu_{e}+{ }^{160} \mathrm{Gd} \rightarrow \rightarrow^{160} \mathrm{~Tb}+e^{-}$
$---\bar{\nu}_{e}+{ }^{156} \mathrm{Gd} \rightarrow{ }^{156} \mathrm{Eu}+e^{+}$
$---\bar{\nu}_{e}+{ }^{158} \mathrm{Gd} \rightarrow{ }^{158} \mathrm{Eu}+e^{+}$
$---\bar{\nu}_{e}+{ }^{160} \mathrm{Gd} \rightarrow{ }^{160} \mathrm{Eu}+e^{+}$

c)

FIG. 2: (Color on line) (a) Total cross sections of $\nu_{e}$ and $\bar{\nu}_{e}$ Gd interactions for each isotope. (b) Total cross sections of $\nu_{e}$ and $\bar{\nu}_{e}$ Gd interactions for each isotope in the energy region from 0 to $15 \mathrm{MeV}$. (c)Total cross sections of natural Gadolinium $G d\left(\nu_{e}, e^{-}\right) T b$ (solid line) and $G d\left(\bar{\nu}_{e}, e^{+}\right) E u$ (dashed line) taking into account the corresponding abundances of each isotope.

for the charged current neutrino(anti-neutrino) scattering leading to final states are shown in Figures 3 (a), (c) and 4(a),(c) for the iotopes ${ }^{155,157} \mathrm{~Tb}\left({ }^{155,157} \mathrm{Eu}\right)$, respectively. It can be seen that the dominant transition is to the final states $1 / 2^{-}, 3 / 2^{-}$and $5 / 2^{-}$. In addition, the final nuclear state distribution in the neutrino-induced reactions is within a wide range of excitation energy 2 to $8 \mathrm{MeV}$. In contrast, for antineutrino scattering, the most prominent transitions are lying between 5 to $7 \mathrm{MeV}$.

The number of expected neutrino events is estimated in a WCD using our predictions of the total cross sections taking $0.1 \%$ (by mass) of Gd doping [23]. In the SK detector where the fiducial mass of water is 32 ktons the 

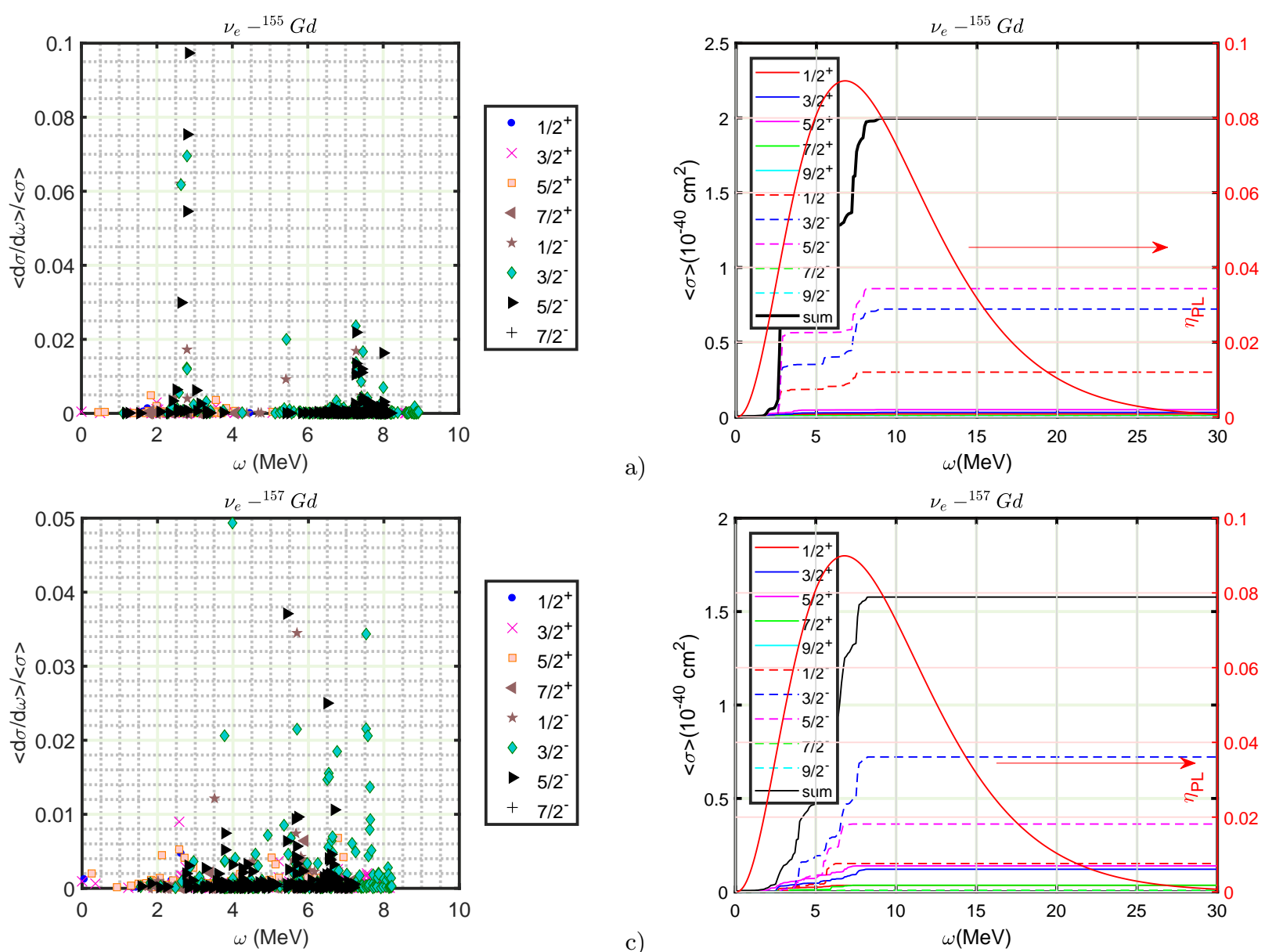

a)

b)
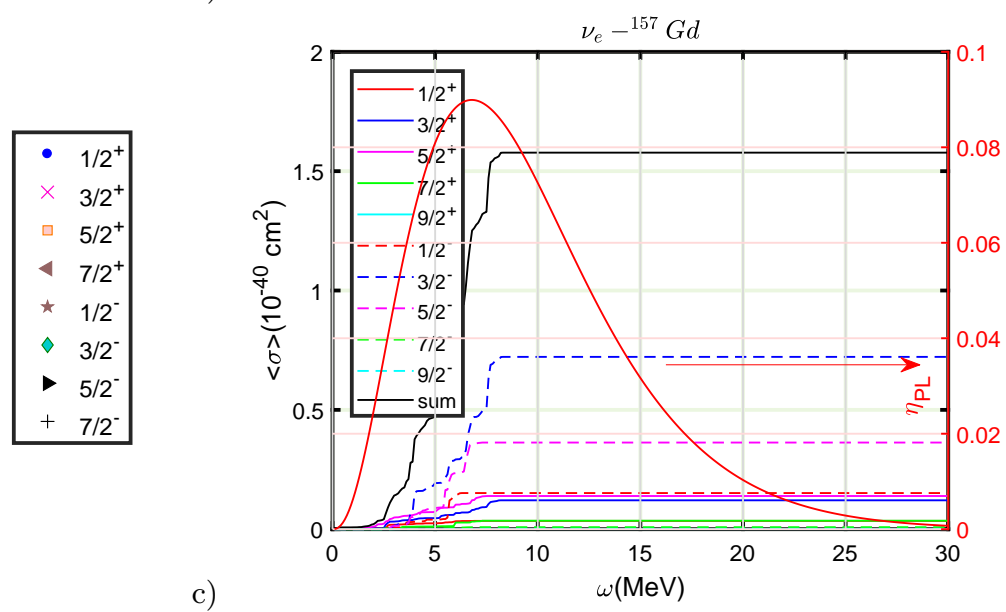

d)

FIG. 3: (Color on line) (a) and (c) Differential cross sections (normalized) for the charged current neutrino interactions off ${ }^{155,157} \mathrm{Gd}$ to final states in ${ }^{155,157} \mathrm{~Tb}$. (b) and (d) Cumulative flux-averaged cross sections (in units $\left.10^{-40} \mathrm{~cm}^{2}\right)$ as a function of excitation energy $\omega$ for the reactions ${ }^{A} G d\left(\nu_{e}, e^{-}\right){ }^{A} T b, \mathrm{~A}=155,157$. The power-law(PL) distributions $\eta_{P L}$ (red solid line) for $\left\langle E_{\nu_{e}}\right\rangle=9.5 \mathrm{MeV}$ and $\alpha=2.5$ is also displayed.

Gadolinium mass there would be about $m_{t}=32$ tons. Supernova radiates $3 \times 10^{53}$ erg of total energy in about $10 \mathrm{~s}$ through neutrinos. Assuming equal energy partition among neutrinos, supernova radiates $N_{\nu_{e}}=3.0 \times 10^{57}$ electron neutrinos and $N_{\bar{\nu}_{e}}=2.6 \times 10^{57}$ electron antineutrinos. The fluence $\Phi\left(E_{\nu}\right)$ for neutrinos integrated over $15 \mathrm{~s}$ burst is given by the relation

$$
\Phi_{i}\left(E_{\nu}\right)=\frac{N_{i}}{4 \pi D^{2}} \eta_{P L}\left(E_{\nu}\right), \quad i=\nu_{e}, \bar{\nu}_{e}
$$

at a distance $D=10 \mathrm{kpc}=3.1 \times 10^{22} \mathrm{~cm}$. Where $\eta_{P L}\left(E_{\nu}\right)$ being the power-law distribution [24, 25]:

$$
\eta_{P L}\left(E_{\nu}\right)=\frac{E_{\nu}^{\alpha} e^{-E_{\nu} / T_{i}}}{T_{i}^{\alpha+1} \Gamma(\alpha+1)}
$$

with

$$
T_{i}=\frac{\left\langle E_{i}\right\rangle}{(\alpha+1)}, \quad i=\nu_{\mathrm{e}}, \bar{\nu}_{\mathrm{e}}
$$

If the mass of the target material is $m_{t}$, corresponding to $N_{t}$ atoms then the number of expected events are

$$
N_{\text {event }}=N_{t} \int \Phi_{i}\left(E_{\nu}\right) \sigma_{i}\left(E_{\nu}\right) d E_{\nu}=N_{t} \frac{N_{i}}{4 \pi D^{2}}\left\langle\sigma_{i}\right\rangle
$$



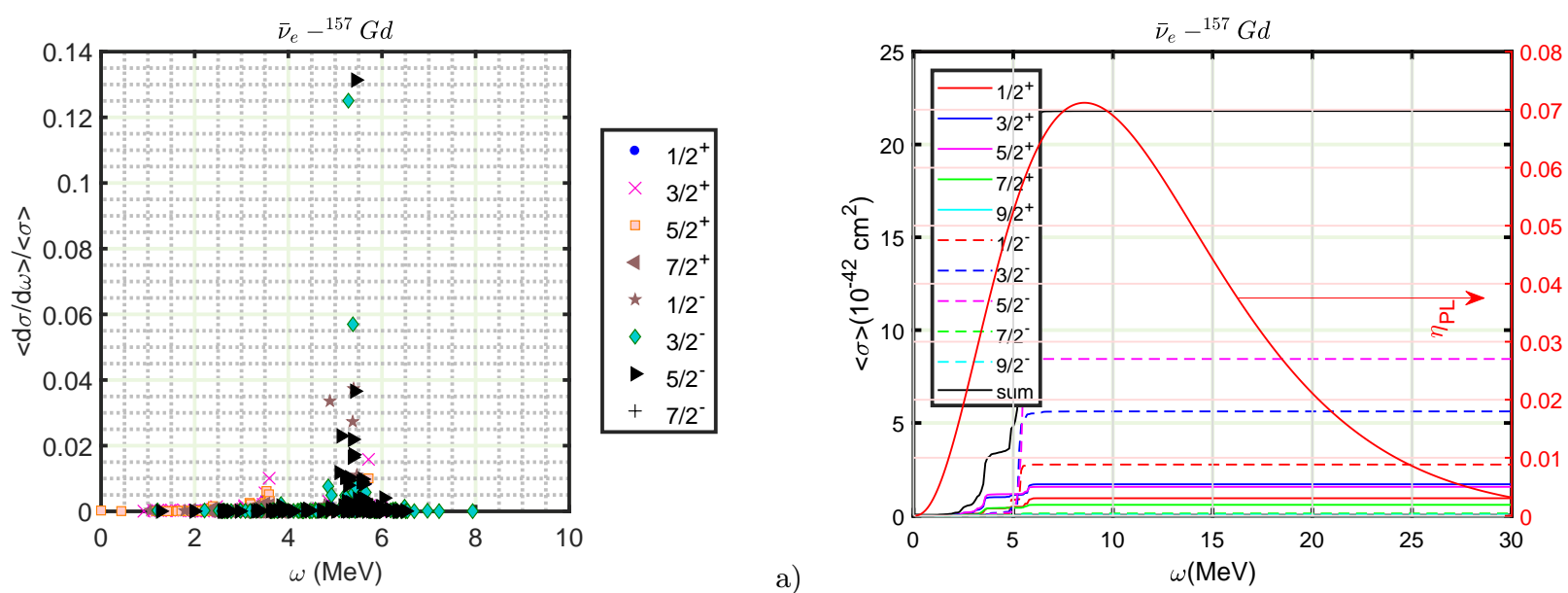

b)

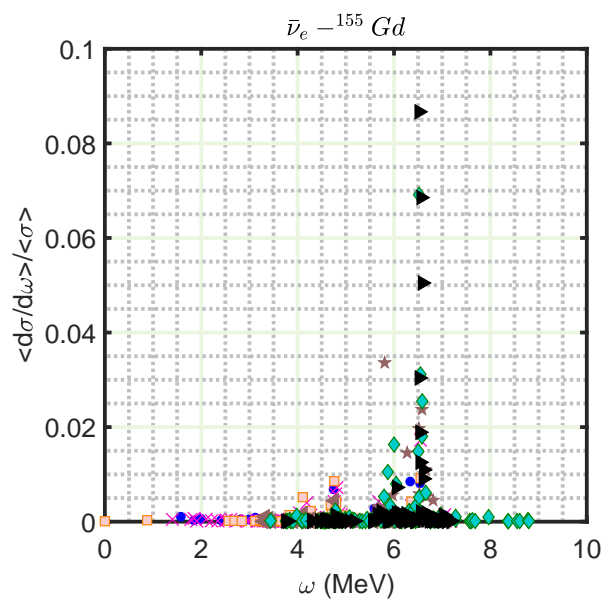

a)

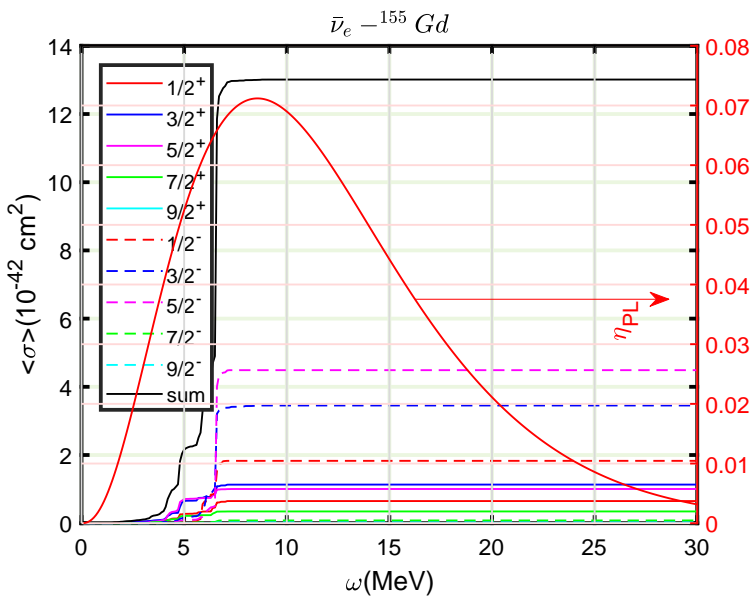

d)

FIG. 4: (Color on line) (a) and (c) Differential cross sections (normalized) for the charged current neutrino interactions off ${ }^{155,157} \mathrm{Gd}$ to final states in ${ }^{155,157} \mathrm{Eu}$. (b) and (d) Cumulative flux-averaged cross sections (in units $\left.10^{-42} \mathrm{~cm}^{2}\right)$ as a function of excitation energy $\omega$ for the reactions ${ }^{A} G d\left(\nu_{e}, e^{-}\right)^{A} E u, \mathrm{~A}=155,157$. The power-law(PL) distributions $\eta_{P L}$ (red solid line) for $\left\langle E_{\nu_{e}}\right\rangle=12 \mathrm{MeV}$ with $\alpha=2.5$ is also displayed.

where $\left\langle\sigma_{i}\right\rangle$ being the flux-averaged cross section.

Neutrinos in water can be detectable through the channels $\bar{\nu}_{e}+p$ (IBD), the elastic scattering $\nu_{e}+e^{-}(\mathrm{ES})$ as well as the neutral current scattering $\nu_{e}+{ }^{16} O(\mathrm{OS})$ on oxygen. As it is known the largest number of events will be due to the IBD which is almost isotropic [1]. Neutrons produced in the IBD are captured by Gd nuclei. These neutrons are easy to see because the Cherenkov light generated by the electrons was struck by a gamma cascade of about $8 \mathrm{MeV}$ emitted by the Gd nucleus. In Fig. [5 a contour plot is used to display the number of expected events for the reactions $G d\left(\nu_{e}, e^{-}\right) T b$ and $G d\left(\bar{\nu}_{e}, e^{+}\right) E u$ respectively, with various parameterizations of power-law spectra. As shown in the figure, in the window of 10-18 MeV, the number of events almost depends on the pinching parameter $\alpha$. Furthermore, the $\bar{\nu}_{e}-\mathrm{Gd}$ events are quite small and are hidden by the large IBD interactions on free protons $[9]$. The ability to completely isolate major IBD events is extremely important for studying the remaining reactions $\nu_{e}-\mathrm{Gd}$ that cause the gamma-ray emission. If $\nu_{e}-\mathrm{Gd}$ events can be isolated by gamma-ray identification or by determining possible delayed beta decay, then they may have certain advantages due to low thresholds (although low yields). Recently it is shown that the Gd-ion release from a custom designed glass in the form of beads or powders could be used as a controlled Gd-source in future WCDs to enhance neutrino detection [26]. 

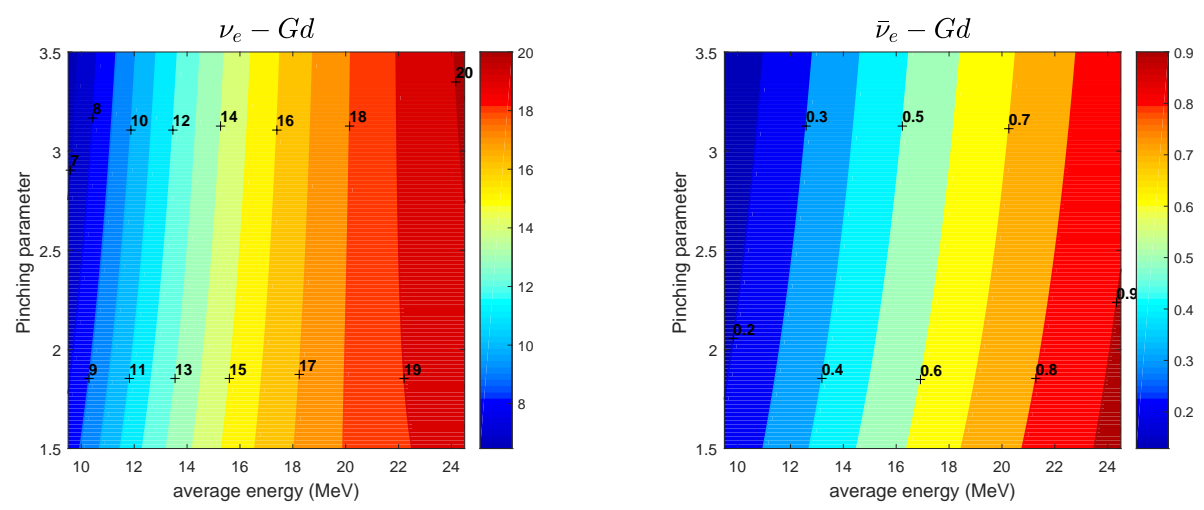

FIG. 5: (Color on line) These contour plots show the number of electrons (left panels) and positrons (right panels) emitted from 32 tons of natural Gd. The contours from left to right in each panel indicates the increase in the number of expected events.

TABLE II: The first column gives the neutrino nucleus reaction and the second column gives the corresponding mean energy $\left\langle E_{\nu}\right\rangle$. The third column gives the total flux-averaged cross sections in units of $10^{-42} \mathrm{~cm}^{2}$. The pinching parameter is taken to be $\alpha=2.5$.

\begin{tabular}{|c|c|c|}
\hline & $\left\langle E_{\nu}\right\rangle(\mathrm{MeV})$ & $\langle\sigma\rangle\left(10^{-42} \mathrm{~cm}^{2}\right)$ \\
\hline \hline$\nu_{e}-{ }^{155} \mathrm{Gd}$ & 9.5 & 199 \\
$\nu_{e}-{ }^{156} \mathrm{Gd}$ & 9.5 & 200 \\
$\nu_{e}-{ }^{157} \mathrm{Gd}$ & 9.5 & 158 \\
$\nu_{e}-{ }^{158} \mathrm{Gd}$ & 9.5 & 224 \\
$\nu_{e}-{ }^{160} \mathrm{Gd}$ & 9.5 & 241 \\
\hline$\nu_{e}-\mathrm{Gd}$ & 9.5 & 205 \\
\hline \hline $\bar{\nu}_{e}-{ }^{155} \mathrm{Gd}$ & 12 & 13.0 \\
$\bar{\nu}_{e}-{ }^{156} \mathrm{Gd}$ & 12 & 13.7 \\
$\bar{\nu}_{e}-{ }^{157} \mathrm{Gd}$ & 12 & 21.8 \\
$\bar{\nu}_{e}-{ }^{158} \mathrm{Gd}$ & 12 & 9.7 \\
$\bar{\nu}_{e}-{ }^{160} \mathrm{Gd}$ & 12 & 8.4 \\
\hline $\bar{\nu}_{e}-\mathrm{Gd}$ & 12 & 12.6 \\
\hline
\end{tabular}

The flux-averaged supernova-neutrino $(S N-\nu)$ cross sections, for the various Gd isotopes, appear in Table 凹. The pinching parameter $\alpha$ has been taken the value $\alpha=2.5$, at a typical supernova neutrino (antineutrino) mean energy $\left\langle E_{\nu_{e}}\right\rangle=9.5 \mathrm{MeV}\left(\left\langle E_{\bar{\nu}_{e}}\right\rangle=12 \mathrm{MeV}\right)$. It can be seen that the cross sections of odd mass isotopes are of the same order of magnitude as the cross sections of even mass isotopes [9]. The cross sections for natural Gd in the reactions $G d\left(\nu_{e}, e^{-}\right) T b\left(20510^{-42} \mathrm{~cm}^{2}\right)$ and $G d\left(\bar{\nu}_{e}, e^{+}\right) E u\left(12.610^{-42} \mathrm{~cm}^{2}\right)$, have been taken from the corresponding abundances of each isotope. The results refer to an ideal detector operating down to zero threshold $E_{t h}=0$. In the case of non zero threshold the flux averaged cross sections it is expected to be suppressed by about $9 \%$ for an electron total energy threshold of $5 \mathrm{MeV}$ (energy threshold in SK)Ref. [9]. 


\section{CONCLUSIONS}

In this article, we have expanded the calculation of Ref. [9] to include the cross-sections of charged current neutrinos and antineutrinos scattering off the odd gadolinium isotopes, ${ }^{155} \mathrm{Gd}$ and ${ }^{157} \mathrm{Gd}$. The neutrino induced transitions to excited nuclear states are computed in the framework of quasiparticle-phonon model. We give the functional relationship between the total cross section and the energy of the neutrino (antineutrino), so that it is possible to obtain the total cross section of the required neutrino energy distribution in the range of 5 to $80 \mathrm{MeV}$. The nuclear responses to supernova neutrinos for the nuclei under consideration have been calculated by folding the cross sections with a power-law quasi-thermal neutrino distribution. The odd mass isotope cross sections we obtained are of the same order of magnitude as the even mass isotope cross sections. The dominant transitions are to the final states $1 / 2^{-}, 3 / 2^{-}$and $5 / 2^{-}$. The final nuclear states in the neutrino-induced reaction are distributed in a wide excitation energy range of 2 to $8 \mathrm{MeV}$, while for antineutrino scattering, the most prominent transitions lie between 5 and $7 \mathrm{MeV}$. The ability to completely isolate the major IBD events is extremely important for studying the remaining reactions

$\nu_{e}-\mathrm{Gd}$ that cause gamma-ray emission. If $\nu_{e}-\mathrm{Gd}$ events can be isolated, then they may have certain advantages due to low thresholds (although the yield is low).

The observation of the next (extra) galactic supernova is very interested in astrophysics and particle physics. Although the uncertainty of neutrino flux is still large, this observation will bring important information about the explosion mechanism of the neutrino light curve, as well as important information about flavor conversion and energy spectrum reconstruction of supernova neutrinos. Megaton-scale water detectors with $\mathrm{GdCl}_{3}$ will have a huge impact on the detection of supernova $\nu_{e}$. They can observe hundreds of diffuse supernova neutrino background events per year, so that they can strictly test the black hole formation rate and supernova neutrino spectrum. 
[1] P. Vogel and J. F. Beacom, Phys. Rev. D 60, 053003 (1999).

[2] A. Strumia and F. Vissani, Phys. Lett. B 564, 42 (2003).

[3] W. M. F. J. F. Beacom and P. Vogel, Phys. Rev. D 66, 033001 (2002).

[4] R. Becker-Szendy et al., Nucl. Instrum. Meth. A 324, 363 (1993).

[5] Y. Fukuda et al., Nucl. Instrum. Meth. A 501, 418 (2003).

[6] A. Bellerive, J. Klein, A. McDonald, A. Noble, and A. Poon, Nucl. Phys. B 908, 30 (2016).

[7] R. Laha and J. F. Beacom, Phys.Rev. D 89, 063007 (2014).

[8] M. R. Vagins, Nucl.Phys.Proc.Suppl. 229-232, 325 (2012).

[9] P. C. Divari, JCAP 09, 325 (2018).

[10] J. Walecka, Theoretical Nuclear and Subnuclear Physics (Oxford University Press, New York, 1995) p. 531 (1995).

[11] T. Donnelly and J. D. Walecka, Nucl. Phys. A 201, 81 (1973).

[12] T. Donnelly and W. Haxton, At. Data and Nucl. Data Tables 23, 103 (1979).

[13] T. Donnelly and R. Peccei, Phys. Rep 50, 1 (1979).

[14] M. Athar, S. Ahmad, and S. Singh, Nucl. Phys. A 764, 551 (2006).

[15] S. Singh, Nucl. Phys. B(Proc. Suppl.) 112, 77 (2002).

[16] P. C. Divari, J. Phys. G: Nucl. Part. Phys. 40, 125201 (2013).

[17] P. Ring and P. Schuck, The Nuclear Many-body Problem (Springer, Berlin, 180).

[18] J. Suhonen, From Nucleons to Nucleus: Concepts of Microscopic Nuclear Theory (Springer, Berlin, 2007).

[19] C. W. Reich, Nuclear Data Sheets 104, 150 (2005).

[20] R. Hemler, Nuclear Data Sheets 78, 219 (1996).

[21] A. Bohr and B. R. Mottelson, Nuclear structure (Benjamin, New York, 1969).

[22] K. Holinde, Phys. Rep. 68, 121 (1981).

[23] J. F. Beacom and M. R. Vagins, Phys. Rev. Lett. 93, 171101 (2004).

[24] C. Lujan-Peschard, G. Pagliaroli, and F. Vissani, JCAP 1407, 051 (2014).

[25] I. Tamborra, B. Muller, L. Hudepohl, H. Janka, and G. Raffelt, Phys. Rev. D 86, 125031 (2012).

[26] R. Dongol and S. Sundaram, Jinst 12, 09028 (2017). 